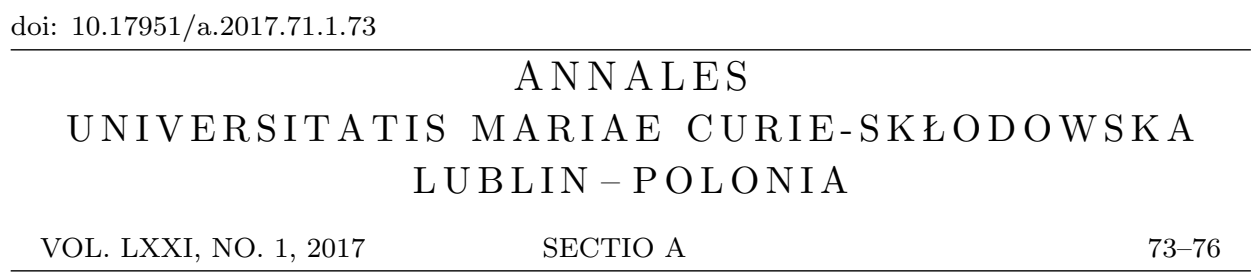

RAYMOND MORTINI

\title{
The Riemann-Cantor uniqueness theorem for unilateral trigonometric series via a special version of the Lusin-Privalov theorem
}

\begin{abstract}
Using Baire's theorem, we give a very simple proof of a special version of the Lusin-Privalov theorem and deduce via Abel's theorem the Riemann-Cantor theorem on the uniqueness of the coefficients of pointwise convergent unilateral trigonometric series.
\end{abstract}

1. Introduction. The earliest uniqueness theorem for trigonometric functions, postulated by Riemann and proved by Cantor reads as follows ([7, p. 326, Theorem 3.1, Chap. IX, Vol. I]):

Theorem 1.1 (Riemann-Cantor). If the trigonometric series $\sum_{n \in \mathbb{Z}} a_{n} e^{i n \theta}$ converges for all $\theta \in \mathbb{R}$ to 0 , then $a_{n}=0$ for all $n \in \mathbb{Z}$.

The proof is, in our viewpoint, rather tricky and technical. It is the aim of this note to give, for the unilateral trigonometric series, a simple, quite elementary proof which is mainly based on Baire's theorem. To achieve our goal, we present a simple proof of a very special case of the Lusin-Privalov theorem [5] on boundary values of functions holomorphic in the disk. For a nice survey on these uniqueness theorems, we refer to [3].

2010 Mathematics Subject Classification. Primary 30H05, 42A20; Secondary 30B30.

Key words and phrases. Boundary behaviour of analytic functions, trigonometric series. 
2. A special case of the Lusin-Privalov uniqueness theorem and the Riemann-Cantor theorem. Let $\mathbb{D}=\{z \in \mathbb{C}:|z|<1\}$ be the unit disk and $\mathbb{T}=\{z \in \mathbb{C}:|z|=1\}$ its boundary. The space of all bounded holomorphic functions in $\mathbb{D}$ is denoted, as usual, by $H^{\infty}:=H^{\infty}(\mathbb{D})$. One of the earliest theorems in function theory of the disk, and which used Lebesgue's theory, stems from Fatou [2] and tells us that every $f \in H^{\infty}$ admits radial limits $f^{*}\left(e^{i t}\right):=\lim _{r \rightarrow 1} f\left(r e^{i t}\right)$ almost everywhere. A short time later, the Riesz brother's [4] showed that if $f^{*}=0$ on a set of positive Lebesgue measure, then $f \equiv 0$. G. Szegö realized that actually $\log \left|f^{*}\right| \in$ $L^{1}(\mathbb{T})$ if $f \not \equiv 0, f \in H^{\infty}$. This can be seen in the following way whenever $f(0) \neq 0$ and $\|f\|_{\infty} \leq 1$ : since $\log |f|$ is subharmonic,

$$
\log |f(0)| \leq \frac{1}{2 \pi} \int_{0}^{2 \pi} \log \left|f\left(r e^{i t}\right)\right| d t .
$$

Now we apply Fatou's lemma to the functions $p_{n}(t)=-\log \left|f\left(r_{n} e^{i t}\right)\right|$, where $r_{n} \rightarrow 1$ is chosen so that $f$ has no zero on the circles of radii $r_{n}$. Thus, Fatou's inequality $\int \liminf p_{n} \leq \liminf \int p_{n}, p_{n} \geq 0$, yields

$$
\log |f(0)| \leq \frac{1}{2 \pi} \int_{0}^{2 \pi} \log \left|f^{*}\left(e^{i t}\right)\right| d t .
$$

We are now ready to prove the "baby" version of the Lusin-Privalov theorem (see also [1, p. 12]):

Theorem 2.1. Let $f$ be holomorphic in $\mathbb{D}$ and suppose that $\lim _{r \rightarrow 1} f\left(r e^{i \theta}\right)=$ 0 for every $\theta \in \mathbb{R}$. Then $f \equiv 0$.

Proof. Consider the set of continuous functions $u_{\theta}:[0,1] \rightarrow \mathbb{C}$ given by $u_{\theta}(r):=f\left(r e^{i \theta}\right)$. Each of these functions is bounded. So

$$
\mathbb{T}=\bigcup_{n=1}^{\infty}\left\{e^{i \theta}:\left|u_{\theta}\right| \leq n \text { on }[0,1]\right\}
$$

is a countable union of closed sets. By Baire's theorem, there is $n_{0}$ such that

$$
\left\{e^{i \theta}:\left|u_{\theta}\right| \leq n_{0} \text { on }[0,1]\right\}
$$

contains an open $\operatorname{arc} I \subseteq \mathbb{T}$. Let $J$ be a closed arc with the same center as $I$ with $J \subseteq I$. Then $f$ is bounded on the sector $S=\{z \in \mathbb{D}: z /|z| \in J\}$ and $f$ has radial limit 0 everywhere on $I$. Let $U:=S^{\circ}$. Using a suitable rotation, we may assume that $U=\{z \in \mathbb{D}: 0<\arg z<\alpha\}$. Map $U$ by a conformal map $\phi$ onto the unit disk; we may take

$$
\phi=\frac{z-i}{z+i} \circ z^{2} \circ \frac{1+z}{1-z} \circ z^{\frac{\pi}{\alpha}} .
$$

Note that $\phi$ has a holomorphic extension to $J^{\circ}$. Let $\tilde{J}=\phi(J)$. Then any ray in $U$ ending at a point in $J$ goes to a simple curve $\gamma$ tending to a point 
on $\tilde{J}$. So $g:=f \circ \phi^{-1} \in H^{\infty}(\mathbb{D})$ and tends to 0 on $\gamma$. By Lindelöf's theorem (for a short, elegant and easy proof, see $[6$, p. 259]) $g$ tends radially to 0 along every radius ending at $\tilde{J}^{\circ}$. Formula (2.1) now implies that $g=f \circ \phi^{-1}$ must be the zero function in $\mathbb{D}$ and so $f \equiv 0$ in $\mathbb{D}$ (note that we do not have to use Fatou's theorem on the existence of radial limits, since it is assumed that $f$ admits these limits and that $\phi^{-1}$ definitely has an analytic extension at all, but three points of $\mathbb{T}$ ).

Here is yet a more elementary approach, communicated to me by Robert Burckel, which does not even use formula (2.1) and the notion of subharmonicity.

We know from the proof above that the function $g \in H^{\infty}$ has radial limits 0 at every point of the open arc $\tilde{J}^{\circ}$. Taking suitable rotations of this arc, we arrive at a function $G(z)=\prod_{j=1}^{m} g\left(e^{i \theta_{j}} z\right), G \in H^{\infty}$, that has radial limit 0 everywhere. Computing the Taylor coefficients $b_{n}$ of $G$ using the formula

$$
b_{n}=\frac{1}{2 \pi} \int_{0}^{2 \pi} G\left(s e^{i t}\right) s^{-n} e^{-i n t} d t, \quad 0<s<1,
$$

we may use Lebesgue's dominated convergence theorem with $s \rightarrow 1$ to get $b_{n}=0$ for every $n \in \mathbb{N}$. Hence $G$ and therefore $f \equiv 0$.

Corollary 2.2 (Unicity theorem). Let $S(\theta) \sim \sum_{n \in \mathbb{N}} a_{n} e^{i n \theta}$ be a (one sided) trigonometric series. Suppose that for every $\theta \in \mathbb{R}$ the series converges to 0 . Then $a_{n}=0$ for every $n \in \mathbb{N}$.

Proof. Associate with $S$ the series $f(z):=\sum_{n \in \mathbb{N}} a_{n} z^{n}$. Since $S(0)=$ $\sum_{n>0} a_{n}$ converges, $a_{n} \rightarrow 0$. Thus the radius of convergence of $f$ is at least 1 . Hence $f \in H(\mathbb{D})$. Abel's theorem implies that

$$
\lim _{r \rightarrow 1} f\left(r e^{i \theta}\right)=S(\theta)=0
$$

for every $\theta$. Hence, by Theorem $2.1, f \equiv 0$ in $\mathbb{D}$ and so $a_{n}=0$ for every $n$.

3. Acknowledgements. I warmly thank Robert Burckel for valuable discussions on the subject of this note.

\section{REFERENCES}

[1] Cima, J., Ross, W., The Backward Shift on the Hardy Space, AMS, Providence, 2000.

[2] Fatou, P., Séries trigonométriques et séries de Taylor, Acta Math. 30 (1906), 335-400.

[3] Kechris, A. S., Set theory and uniqueness for trigonometric series, Preprint 1997. http://www.math.caltech.edu/ kechris/papers/uniqueness.pdf

[4] Riesz, F., Riesz, M., Über die Randwerte einer analytischen Funktion, Quatrième Congrès des Math. Scand. (1916), 27-44,

[5] Lusin, N., Privaloff, J., Sur l'unicité et la multiplicité des fonctions analytiques, Ann. Sci. ENS 42 (1925), 143-191.

[6] Rudin, W., Real and Complex Analysis, third edition, McGraw-Hill, New York, 1986. 
[7] Zygmund, A., Trigonometric Series, second edition, Vol. I+II Combined, Cambridge Math. Lib. 1959 and 1993.

Raymond Mortini

Université de Lorraine

Département de Mathématiques et

Institut Élie Cartan de Lorraine, UMR 7502

Ile du Saulcy

F-57045 Metz, France

e-mail: raymond.mortini@univ-lorraine.fr

Received October 16, 2016 